\title{
Optical intersubband transitions in quantum wires with an applied magnetic field
}

\author{
G.B. Ibragimov \\ Institute of Physics, NAS of Azerbaijan Republic, 33, Javid av., 1143 Baku, Azerbaijan \\ E-mail: guseyn@physics.ab.az,guseyn_gb@mail.ru
}

\begin{abstract}
The intersubband optical absorption is investigated in parabolic quantum wires in the presence of a tilted magnetic fields. We show that for increasing magnetic field the intersubband absorption peak is shifted to higher energies and its amplitude is increased, too. In particular, it has been shown that the direction of the magnetic field plays a significant role in the intersubband optical absorption.
\end{abstract}

Keywords: quantum wire, optical intersubband transitions.

Paper received 23.09.03; accepted for publication 21.10.04.

\section{Introduction}

During the past three decades, the physics of low-dimensional semiconductors has become a vital part of presentday research. Low-dimensional structures allow the study of a variety of new mechanical, optical and transport phenomena. In this context, one-dimensional systems have been of particular interest for past decade.

There is a considerable interest in using the intersubband transitions in low-dimensional systems. Intersubband optical transition has attracted considerable attention. It occurs between the subbands that are created within the same band: its wavelength ranges mostly from near to far infrared. This latter transition suggests the possibility of a whole new field of applications and related physics. This follows from the unusual features which the above transition is thought to passes: large absorption coefficient [1], narrow absorption linewidth [2], large optical nonlinearity [3], fast intraband relaxation [4] and reduced Auger effect [5]. These features could all be readily exploited to enhance the performance of optoelectronic devices such as lasers, detectors and optical switches.

The improvements of the semiconductor growth techniques have offered the possibility to obtain low-dimensional semiconductor structures with any desired well shapes. One of those structures is the so-called parabolic quantum well. Theoretically, parabolic confining potentials are very attractive, since the spectrum and wave functions of one-electron states have a simple analytical form, it is possible to derive explicit analytical expressions for the coefficient of absorption of high-frequency electromagnetic field. The intersubband optical absorption in low-dimensional structures is well developed both in the absence [6-11] and in the presence of magnetic fields [12-19].

The magnetic field is an interesting additional parameter, since it can be applied experimentally in a wellcontrolled way and modifies fundamentally the electronic structure. The application of a magnetic field to a crystal changes the dimensionality of electronic levels and leads to a redistribution of a density of states. The magnetic field is assumed to be tilted with respect to the normal, it serves to add an extra confining potential to the initial confinement, gives rise to two different kinds of Landau level indices, and causes a dramatic change in the energy spectrum, leading to the so-called hybrid magnetoelectric quantization .

This paper reports the absorption coefficients due to intersubband optical transition in parabolic quantum wires in tilted magnetic fields.. In accordance with the generalized Kohn theorem [20], electron-electron interactions have no effect on the electron transitions in this case.

\section{Formalism}

We consider the transport of an electron gas in a Q1D electron quantum wire structure as treated in [21,22], in which a Q1D electron gas is confined by two confine- 
ment frequencies $\omega_{1}$ and $\omega_{2}$ in the $x$ and $z$ directions, respectively, and the conduction electrons are free along only one direction ( $y$ direction) of the wire. Considering the magnetic field transverse tilt direction, $H=\left(H_{x}, 0\right.$, $H_{z}$ ) with the Landau gauge, the one-electron Hamiltonian $H_{e}$, eigenstates $\Psi_{n l k}$ and eigenvalues $E_{n l k}\left(k_{y}\right)$ are written as $[21,22]$

$$
\begin{aligned}
& H=\frac{1}{2 m^{*}}\left(p+\frac{e A}{c}\right)^{2}+\frac{1}{2} m^{*} \omega_{1}^{2} x^{2}+\frac{1}{2} m^{*} \omega_{2}^{2} z^{2} \\
& \psi_{n l k_{y}}=\left(1 / L_{y}\right)^{1 / 2} \phi_{n}\left(x-x_{0}\right) \phi_{l}\left(z-z_{0}\right) \exp \left(i k_{y} y\right) \\
& E_{n l}\left(k_{y}\right)=(n+1 / 2) \hbar \Omega_{1}+(l+1 / 2) \hbar \Omega_{2}+\frac{p_{y}^{2}}{2 \tilde{m}}
\end{aligned}
$$

where $p$ is the momentum operator of a conduction electron, $\omega_{x}=e H_{x} / m^{*} c=\omega_{c} \cos \vartheta, \omega_{z}=e H_{z} / m^{*} c=\omega_{c} \sin \vartheta$, $\Omega_{1}^{2}=\omega_{1}^{2}+\omega_{z}^{2}, \Omega_{2}^{2}=\omega_{2}^{2}+\omega_{x}^{2}, x_{0}=-b_{1} l_{B 1}^{2} k_{y}, z_{0}=b_{2} l_{B 2}^{2} k_{y}$ and $\tilde{m}=m^{*}\left(\Omega_{1}^{2} \Omega_{2}^{2}\right)\left(\omega_{1}^{2} \omega_{2}^{2}-\omega_{x}^{2} \omega_{z}^{2}\right)^{-1}$. Here $l_{B 1}=$ $=\left(\hbar / m^{*} \Omega_{1}\right)^{1 / 2}, l_{B 2}=\left(\hbar / m^{*} \Omega_{2}\right)^{1 / 2}, b_{1}=\omega_{z} / \Omega_{1}, b_{2}=$ $=\omega_{x} / \Omega_{2}$ and $\phi_{n, l(x)}$ represent harmonic-oscillator wave functions. For the sake of simplicity, we assume that the coupling term $H_{x} H_{z} x z$ in Eq. (1) is negligible [22,23] since its contribution to the total electron energy in these systems is minor.

The absorption coefficient for the case of nondegenerate electron gas in first-order perturbation theory is given by $[12,13,24]$

$$
\begin{aligned}
& \alpha=\frac{2 \pi \sqrt{\epsilon(\omega)}}{c \hbar n_{0}}\left[1-\exp \left(-\frac{\hbar \omega}{K_{B} T}\right)\right] \times \\
& \times\left.\sum_{n l k_{y}} \sum_{n l k_{y}} f_{0}\left(E_{n l k_{y}}\right)\left\langle n l k_{y}\left|H_{R}\right| n^{\prime} l^{\prime} k_{y}^{\prime}\right\rangle\right|^{2} \times \\
& \times \delta\left(E_{n l k_{y}}-E_{n^{\prime} l^{\prime} k_{y}^{\prime}}+\hbar \omega\right),
\end{aligned}
$$

where $\varepsilon(\omega)$ is the real part of the dielectric constant, $n_{0}$ the number of photons in the unit volume with frequency $\omega, \mathrm{c}$ is the speed of light in vacuum, $\left[1-\exp \left(-\hbar \omega / K_{B} T\right)\right]$ gives the emission of the photons and $H_{R}$ is the interaction Hamiltonian between electrons and the radiation field. The electron distribution function $f_{0}\left(E_{n l k}\right)$ for quasione-dimensional nondegenerate electron gas in the presence of a magnetic field can be shown as

$$
\begin{aligned}
& f_{0}=\frac{4 \sqrt{2} \hbar N \sinh \left(\hbar \Omega_{1} / 2 K_{B} T\right) \sinh \left(\hbar \Omega_{2} / 2 K_{B} T\right)}{L_{y} \sqrt{\tilde{m} K_{B} T}} \times \\
& \times \exp \left(-E_{n l k_{y}} / K_{B} T\right) .
\end{aligned}
$$

We write the Hamiltonian $H_{R}$ representing the interaction with the high-frequency field in the form

$$
H_{R}=\frac{e}{m^{*}} \sqrt{\frac{2 \pi \hbar n_{0}}{\in(\omega) \omega}} \varepsilon\left(p+\frac{e}{c} A\right),
$$

where $\varepsilon$ is the polarization vector of the radiation field. In the calculation of the matrix elements of $H_{R}$ that follow, the high-frequency field is assumed uniform. For this, the photon wavelength $\lambda$ must be much larger than $l_{1,2}$, which imposes certain restrictions on the hybridoscillation frequencies $\Omega_{1,2}$. If this condition is met, the electron-photon transitions are the dipole ones. Below we shall calculate the absorption for linear polarization and choose the polarization vector $\varepsilon$ in the $y$ direction. Then the matrix element of the electron-photon interaction Hamiltonian can be written as

$$
\begin{aligned}
& \left\langle n l k_{y}\left|H_{R}\right| n^{\prime} l^{\prime} k_{y}^{\prime}\right\rangle= \\
& =\frac{e}{m^{*}} \sqrt{\frac{2 \pi \hbar n_{0}}{\in(\omega) \omega}}\left\langle n l k_{y} \mid\left(P_{y}+x m^{*} \omega_{z}-z m^{*} \omega_{x}\right) n^{\prime} l^{\prime} k_{y}^{\prime}\right\rangle
\end{aligned}
$$

A straightforward calculation of the matrix element square in the representation (2) gives

$$
\begin{aligned}
& \left|\left\langle n l k_{y}\left|H_{R}\right| n^{\prime} l^{\prime} k_{y}^{\prime}\right\rangle\right|^{2}=\left(\frac{2 \pi \hbar n_{0}}{\in(\omega) \omega}\right)\left(\frac{e^{2}}{b_{1}^{2}} \omega_{z}^{2} x_{0}^{2} \delta_{n n^{\prime}} \delta_{l l^{\prime}} \delta_{k_{y} k_{y}^{\prime}}+\right. \\
& +\left(\frac{e \omega_{z} l_{b 1}}{\sqrt{2}}\right)^{2}\left[n \delta_{n^{\prime} n-1}+(n+1) \delta_{n^{\prime} n+1}\right] \delta_{l l^{\prime}} \delta_{k_{y} k_{y}^{\prime}}+ \\
& \left.+\left(\frac{e \omega_{x} l_{b 2}}{\sqrt{2}}\right)^{2}\left[l \delta_{l l-1}+(l+1) \delta_{l l+1}\right] \delta_{n n^{\prime}} \delta_{k_{y} k_{y}^{\prime}}\right\}
\end{aligned}
$$

where Kronecker symbols $\left(\delta_{n n^{\prime}}, \delta_{l l^{\prime}}, \delta_{k_{y} k_{y}^{\prime}}\right)$ denote the selection rules, which arise during the integration of the matrix element with respect to each direction.

Plugging (5) and (8) into (4), we can further perform the sum over $n(l)$ by writing $\sum n \exp (-\alpha n)=$

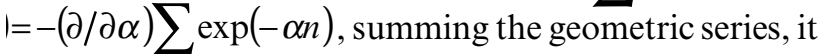
is found that the absorption coefficient for quasi-one-dimensional electron gas is given by

$$
\begin{aligned}
& \alpha=\frac{16 \pi^{3 / 2}\left[1-\exp \left(-\hbar \omega / K_{B} T\right)\right] N e^{2}}{c \sqrt{\epsilon(\omega)} \hbar \omega} \times \\
& \times\left\{\frac{\omega_{z}^{2} x_{0}^{2}}{b_{1}^{2}} \delta(\omega)+\left(\frac{\omega_{z} l_{B 1}}{\sqrt{2}}\right)^{2}\left[\frac{\delta\left(\omega+\Omega_{1}\right)}{\exp \left(\hbar \Omega_{1} / K_{B} T\right)-1}+\right.\right. \\
& \left.+\frac{\exp \left(\hbar \Omega_{1} / K_{B} T\right) \delta\left(\omega-\Omega_{1}\right)}{\exp \left(\hbar \Omega_{1} / K_{B} T\right)-1}\right]+\left(\frac{\omega_{x} l_{B 2}}{\sqrt{2}}\right)^{2} \times \\
& \left.\times\left[\frac{\delta\left(\omega+\Omega_{2}\right)}{\exp \left(\hbar \Omega_{1} / K_{b} T\right)-1}+\frac{\exp \left(\hbar \Omega_{2} / K_{B} T\right) \delta\left(\omega-\Omega_{2}\right)}{\exp \left(\hbar \Omega_{2} / K_{b} T\right)-1}\right]\right\} .
\end{aligned}
$$

To account for the smearing of the hybrid-oscillation resonance, we must replace in $\mathrm{Eq}$. (9) the delta function by the Lorentzian $\delta_{\tau}(x)=(\pi \tau)^{-1} /\left(\tau^{-2}+x^{2}\right)$. In this case, $\alpha(\omega)$ has delta - function - like spikes with a halfwidth equal to $\tau^{-1}$, where $\tau$ is the phenomenological relaxation time. 


\section{G.B. Ibragimov: Optical intersubband transitions in quantum wires with ...}

In the limit that $\hbar \Omega_{1,2}>>K_{B} T$ and $\exp \left(-\hbar \omega / K_{B} T\right)<<$ $<<1$ and $\exp \left(-\hbar \Omega_{1,2} / K_{B} T\right)<<1$, we obtain

$$
\frac{\alpha}{\alpha_{0}}=\frac{8 \pi^{1 / 2} \omega_{c}^{2}}{\omega}\left\{\frac{\sin ^{2} \vartheta}{\Omega_{1}} \frac{1}{1+\tau^{2}\left(\omega-\Omega_{1}\right)}+\right.
$$$$
\left.+\frac{\cos ^{2} \vartheta}{\Omega_{2}} \frac{1}{1+\tau^{2}\left(\omega-\Omega_{2}\right)}\right\}
$$

where $\left(\alpha_{0}=e^{2} N \tau / \sqrt{\in(\omega)} \mathrm{cm}{ }^{*}\right)$. From Eq. (10) we see that the intersubband optical absorption shows the resonant behavior at $\omega=\Omega_{1,2}$. We call this resonance at points $\omega=\Omega_{1,2}$, a hybrid resonance. Since the hybrid-oscillator frequencies $\Omega_{1,2}$ depend on the magnitude of the magnetic field and the direction of the magnetic field, the position of the peaks on the $\alpha$ vs $\omega$ curve depends on these parameters.

\section{Results and discussion}

Let as study the absorption coefficient more closely. The detailed description of the absorption peaks requires numerical studies of the $\alpha$ dependence on the radiation frequency, the magnetic field strength, and the field orientations.

In Fig. 1 for $B=7.5 T$, we present the variation of the absorption coefficient $\alpha / \alpha_{0}$ as a function of the photon frequency. The first group of peaks corresponding to the electron transition between the Landau-level states with frequency $\Omega_{1}$, while the second group peaks corresponding the electron transition between the Landau-level states with frequency $\Omega_{2}$. By changing the direction of the magnetic field, we can tune the resonance photon energy for this transition. As seen in this figure for first group peaks, the absorption coefficient increases as the tilt angle increases. Since the confinement $\left(\Omega_{1}\right)$ of the subbands increases with large $\vartheta$ values, the overlap be-

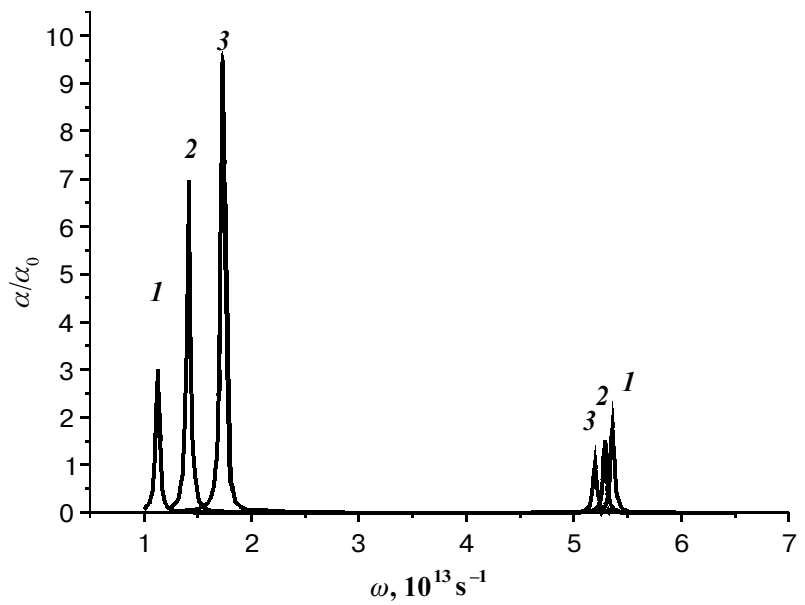

Fig. 1. The intersubband absorption coefficient $\alpha / \alpha_{0}$ vs $\omega$ at various tilt angles $\vartheta$ for the quasi-one-dimensional electron gas ( $\tau=$ $=5 \cdot 10^{12} \mathrm{~s}, \omega_{1}=10^{13} \mathrm{~s}, \omega_{2}=5 \cdot 10^{13} \mathrm{~s}$, are used) for $H=7.5 T$. The lines are, $\vartheta: 1-15^{\circ}, 2-30^{\circ}, 3-45^{\circ}$.

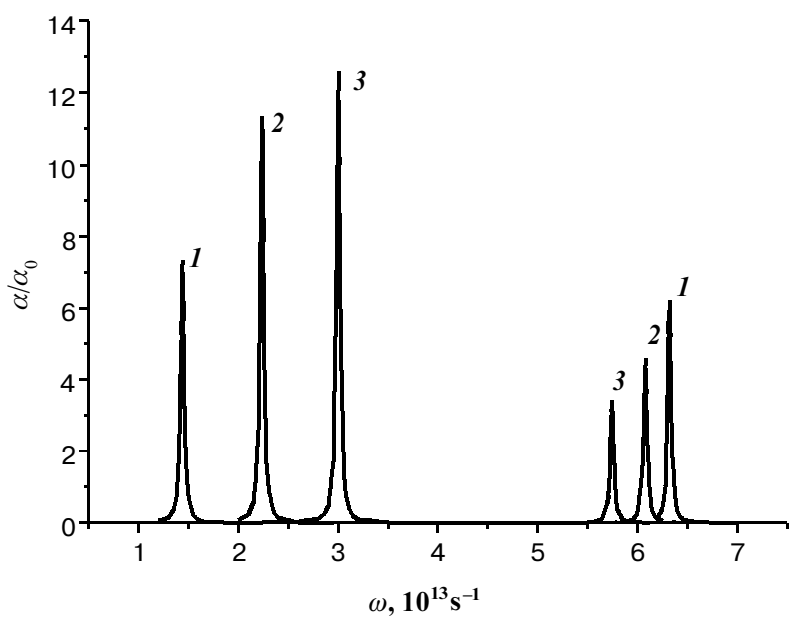

Fig. 2. The intersubband absorption coefficient $\alpha / \alpha_{0}$ vs $\omega$ at various tilt angles $\vartheta$ for the quasi-one-dimensional electron gas $\left(\tau=5 \cdot 10^{12} \mathrm{~s}, \omega_{1}=10^{13} \mathrm{~s}, \omega_{2}=5 \cdot 10^{13} \mathrm{~s}\right.$, are used) for $H=15 T$. The lines are for $\vartheta: 1-15^{\circ}, 2-30^{\circ}, 3-45^{\circ}$.

tween electron subbands increases, thus the magnitude of the absorption coefficient becomes larger. The resonance photon frequency increases with tilt angle $\vartheta$. As seen in this figure the direction of the field is tunable parameter in intersubband optical transitions. This gives an additional degree of freedom in optical device applications.

For the second group absorption peaks, separation between Landau-level states is decreased with increasing tilt angle. Therefore, in this case intersubband optical absorption is decreased in energy with increasing tilt angle. Since the confinement $\left(\Omega_{2}\right)$ of the subbands decreases with large $\vartheta$ values, the overlap between electron subband decreases, thus the magnitude of the absorption coefficient becomes small.

In Fig. 2, we present the absorption coefficient $\alpha / \alpha_{0}$ for the intersubband transition as a function of the incident photon frequency for $H=15 T$. By comparing Fig. 1 with Fig. 2, we find that the resonance photon frequency for intersubband transition increases with the increasing magnetic field. The absorption peak for the intersubband optical absorption is increased in its magnitude with the increasing magnetic field.

It should be noted that in [18] it has been observed experimentally that the photon energy and intensity of the absorption peaks depends on magnetic fields. In [14] also found for the case of two-dimensional system was that the resonance photon frequency increases with the tilt angle $\vartheta$.

In conclusion, interssubband optical absorption in a quantum wire has been studied under an external tilted magnetic field. It is found that the absorption peak is shifted in its energy and is also increased in its magnitude with the increasing magnetic field. We show that the intersubband optical absorption is sensitive to the tilt angle. 


\section{G.B. Ibragimov: Optical intersubband transitions in quantum wires with ...}

\section{Acknowledgments}

The author would like to thank Prof. M. I. Aliev and Prof. F. M. Gashimzade for helpful discussions.

\section{References}

1. L. C. West and S. J. Eglash. First observation of an extremely large-dipole infrared transition withinthe conduction band of a GaAs quantum well // Appl.Phys.Lett., 46, p.1156-1158 (1985).

2. A. Harwit and. J. S. Harris Observation of Stark shifts in quantum well intersubband transition // J. Appl. Phys. Lett., 50, p.685-687 (1987).

3. S.Y Yuen Phase matching in frequency mixing with internally generated waves // Appl.Phys.Lett., 43, p. 813-815 (1987).

4. A. Seilmeier, H.J. Hubner, G. Abstreiter, G. Weiman and W. Schlapp. Intersubband relaxation in $\mathrm{GaAs}-\mathrm{Al}_{\mathrm{x}} \mathrm{Ga}_{1-\mathrm{x}} \mathrm{As}$ quantum well structures observed directly by an infrared bleaching technique // Phys.Rev. Lett., 59, p. 1345-1348 (1987).

5. Perng-fei Yuh and. K.L. Wang. Intersubband Auger recombination in a superlattice // Phys. Rev. B, 37, p. 1328-1333 (1988).

6. E.R. Brown and S.J. Eglash. Calculation of intersubband absorption strength in ellipsoidal-valley quantum wells // Phys. Rev. B, 41, p. $7559-7564$ (1990).

7. Chan-Lon Yang, Des-Son Pan and Robert Somoano Advantages of an indirect semiconductor quantum well system for infrared detection // J. Appl. Phys., 65, p. 3253-3258 (1989).

8. G.G. Zegrya and V.E. Perlin. Intraband light absorption in quantum wells at the expense of electron-electron collisions // Fiz. Tekh. Poluprovodn., 32, p. 466-471 (1998).

9. L.E. Golub, E.L. Ivchenko, R.Ya. Rasulov. Intersubband absorption of light in a semiconductor quantum well with complicated band structure // Fiz. Tekh. Poluprovodn., 29, p. 1093-1100 (1995).

10. Intersubband Transitions in Quantum Wells, ed. by E.Rosencher, B. Vinter, B. Levine (N.-Y.1992)
11. G.B. Ibragimov // J.Phys:Condens. Matter., 14, p. 4977 (2002).

12. G.B. Ibragimov // J.Phys:Condens. Matter., 14, p. 8145 (2002).

13 G.B. Ibragimov, Theory of the free-carrier absorption in quantum wires with boundary roughness scattering // Semiconductor Physics, Quantum Electronics\& Optoelectronics, 6, p. 9-13 (2003).

12. N G Galkin, V. A. Margulis, and A. B. Shoroxov. Intraband absorption of electromagnetic radiation by quantum nanostructures with parabolic confinement potential // Fiz. Tverd. Tela, 43, p. $511-519$ (2001).

13. V. A. Margulis. A hybrid-phonon resonance in a quasi-twodimensional nanostructure // JETP, 111, p. $1092-1106$ (1997)

14. Kasapoglu E., Sari H., Sokmen I. Intersubband optical absorption in a quantum well under a tilted magnetic field // Superlat. and Microstruct., 29, p. 25-32 (2001).

15. E.P. Sinyavskii,S.M. Sokovnich, Intraband absorption of light in quasi-two-dimensional systems in external electric and magnetic fields // Fiz. Tekh. Poluprovodn., 33, p. 828-831 (1999).

16. A.G.Petrov, A.Ya. Shik Interband optica transitions in quantum wells. // Fiz. Tekh. Poluprovodn., 27, p. 1047-1057 (1993).

17. G.B. Ibragimov Free-carrier magnetoabsorption in quantum well structures // Ukr. J. Phys., 48, (6), p. 527-532 (2003).

18. N.Miura, Y.H Matsuda, K. Uchida and H. Arimoto. Magneto-optical study of semiconductor nanostructures in high magnetic fields // J. Phys: Condens. Matter. 11, p. 59175928 (1999).

19. L. Wendler, V.G. Grigoryan, Theory of magneto-optical absorption of the quasi-one-dimensional electron gas // Physica B, 245, p. 127-156 (1998).

20. L. Brey, N.E. Johnson and B.J. Halperin. Optical and magneto-optical absorption in parabolic quantum wells // Phys. Rev. B, 40, p. 10647-10649 (1989).

21. G. Ihm, M.L. Falk, S.K. Noh and J.I. Lee. Oscillating magnetization of quantum wells and wires in tilted magnetic fields // Phys. Rev. B., 46, p. 15530-15533 (1992).

22. Jai Yon Ryu, G.Y. Hu and R.F. O'Connell. Magnetophonon resonances of quantum wires in tilted magnetic fields // Phys. Rev. B, 49, p. 10437-10443 (1994).

23. G. Marx and R. Kummel J.Phys.:Condens. Matter., 3, p. 8237 (1991).

24. F.G. Bass, I.B. Levinson Cyclotron-Phonon Resonance in semiconductoes. // JETP 49, p. 914-924 (1965). 